05,11

\title{
Фазовые переходы в геликоидальных ферромагнетиках с концентрационными флуктуациями локальной намагниченности
}

\author{
(C) А.А. Повзнер, А.Г. Волков
}

Уральский федеральный университет им. Б.Н. Ельцина,

Екатеринбург, Россия

E-mail: a.a.povzner@urfu.ru, agvolkov@yandex.ru

Поступила в Редакцию 21 февраля 2019 г.

В окончательной редакции 21 февраля 2019 г.

Принята к публикации 26 февраля 2019 г.

\begin{abstract}
Развивается феноменологический подход к теории индуцированных флуктуациями фазовых переходов в геликоидальных ферромагнетиках с концентрационными флуктуациями. Для этого в функционал Гинзбурга-Ландау вводятся случайные переменные, значение которых равно единице на узле $v$, занятом магнитным атомом, и нулю - в противном случае. Показано, что выше температуры магнитного перехода $\left(T_{C}\right)$, вследствие концентрационных эффектов сохраняется локальная намагниченность, и возникают флуктуации геликоидальной спиновой спирали, а в магнитном поле формируются скирмионные состояния. Исчезновение вихревых состояний обусловлено подавлением локальной намагниченности термодинамическими флуктуациями при температуре $T_{S}\left(>T_{C}\right)$. Теоретические результаты объясняют причины значительного расширения температурной области скирмионных состояний в нестехиометрическом моносилициде марганца с дефицитом марганца.
\end{abstract}

Ключевые слова: функционал, геликоидальные ферромагнетики, флуктуации, скирмионы.

DOI: 10.21883/FTT.2019.07.47834.387

\section{1. Введение}

Из флуктуационной теории фазовых переходов известно, что увеличение, при приближении к критической области, числа флуктуационных мод и возникновение больших по амплитуде спиновых флуктуаций индуцирует замену перехода второго рода на переход первого рода [1-3]. В результате изменения рода фазового перехода, корреляционная длина становится конечной в точке перехода (не расходится), а параметры порядка не меняются непрерывно. Именно такая ситуация, повидимому, имеет место при фазовом переходе в $\mathrm{MnSi}$, где антисимметричное релятивистское взаимодействие Дзялошинского-Морийя (ДМ-взаимодействие), приводит к возникновению в области дальнего порядка геликоидальной спиновой спирали [1]. В работе [4] было показано, что наблюдаемые при индуцированном флуктуациями фазовом переходе первого рода аномалии теплоемкости и восприимчивости $\mathrm{MnSi}$ возникают в интервале температур, соответствующем изотропным киральным флуктуациям. Согласно нейтронографическим данным длина спиновой когерентности в этой области имеет промежуточное значение между характерными для модели Янсена-Бака (анизотропные киральные флуктуации) и для ферромагнитных флуктуаций [4]. При этом согласие нейтронографических данных с результатами моделирования геликоидального ферромагнетизма с ДМ-взаимодействием в методе Монте-Карло [5,6] достигается после учета продольных флуктуаций магнитных моментов на узлах [6].
Отметим также, что в не стехиометрических образцах $\mathrm{MnSi}$ с заметным дефицитом марганца имеет место увеличение почти на порядок температурного интервала скирмионной фазы по сравнению со стехиометрическим составом [7]. Поэтому концентрационным флуктуациям модуля локальной намагниченности, возникающие при заметных отклонениях от стехиометрии, могут оказаться решающими для формирования вихревых структур в области фазового перехода первого рода, что требует отдельного рассмотрения.

\section{2. Уравнения магнитного состояния}

Рассмотрим функционал Гинзбурга-Ландау $(\Psi(\xi))$ для геликоидального ферромагнетика с ДМ-взаимодействием [4]. В q-представлении этот функционал имеет вид

$$
\begin{aligned}
& \Psi\left(\xi_{\mathbf{q}}\right)=\tau \sum_{\mathbf{q}}\left|\xi_{\mathbf{q}}\right|^{2}+A \sum_{\mathbf{q}} \mathbf{q}^{2}\left|\xi_{\mathbf{q}}\right|^{2} \\
& +\Gamma^{0} \sum_{\mathbf{q}_{1}+\mathbf{q}_{2}+\mathbf{q}_{3}+\mathbf{q}_{4}=0} \xi_{\mathbf{q}_{1}} \xi_{\mathbf{q}_{2}} \xi_{\mathbf{q}_{3}} \xi_{\mathbf{q}_{4}}-i d \sum_{\mathbf{q}} \mathbf{q}\left[\xi_{\mathbf{q}} \xi_{-\mathbf{q}}\right]
\end{aligned}
$$

Здесь, $\xi_{\mathbf{q}}-$ вектор параметра порядка, $\tau=$ $=a\left(1-T_{C}^{(0)}\right)[4,5], T-$ температура в энергетических единицах.

Для рассмотрения наряду с термодинамическими флуктуациями концентрационных флуктуаций, в функ- 
ционале (1) осуществим замены

$$
\xi_{v} \rightarrow p_{v} \xi_{v} .
$$

Здесь $p_{\boldsymbol{v}}$ - случайные переменные, равные единице на узле $v$ занятом магнитным атомом, и нулю - в противном случае.

В записи слагаемого, ответственного за взаимодействие Дзялошинского-Мория, вследствие релятивистской малости этого взаимодействия, воспользуемся приближением среднего поля

$$
\sum_{\boldsymbol{v} \boldsymbol{\mu}} p_{\nu} p_{\mu} \mathbf{d}_{\boldsymbol{\nu} \boldsymbol{\mu}}\left[\xi_{\boldsymbol{v}} \times \xi_{\boldsymbol{\mu}}\right] \approx i d x^{2} \sum_{\mathbf{q}} \mathbf{q}\left[\mathbf{M}_{q} \times \mathbf{M}_{-\mathbf{q}}\right] .
$$

Уравнение магнитного состояния для параметра порядка $\mathbf{M}_{\mathbf{q}}$ (соответствующего среднему значению $\boldsymbol{\xi}$ ) запишем с учетом термодинамических флуктуаций, описываемых в приближении Бразовского [3], и стохастических концентрационных флуктуаций локальной намагниченности $\left(\delta p_{\boldsymbol{v}} \xi_{\boldsymbol{v}}\right)$,

$$
\xi_{\boldsymbol{v}}=x\left(\xi_{\boldsymbol{v}}-\mathbf{M}_{\boldsymbol{v}}\right)+\delta p_{\boldsymbol{v}} \xi_{\boldsymbol{v}}
$$

где $\mathbf{M}_{\boldsymbol{v}}$ - средний вектор параметра порядка на узле $\boldsymbol{v}, x=N_{0}^{-1} \Sigma_{\boldsymbol{v}} p_{\boldsymbol{v}}-$ концентрация магнитных атомов, $\delta p_{\boldsymbol{v}}=p_{\boldsymbol{v}}-x, N_{0}-$ число узлов кристаллической решетки, отвечающих положению магнитных атомов в стехиометрическом случае, $\langle(\ldots)\rangle=Z^{-1} \int(d) \xi_{\mathbf{q}}(\ldots)$ $\times \exp \left(-\Psi\left(\xi_{\mathbf{q}}\right)\right), Z=\int\left(d \xi_{\mathbf{q}}\right) \exp \left(-\Psi\left(\xi_{\mathbf{q}}\right)\right)-$ статистическая сумма.

Уравнения магнитного состояния запишем, используя термодинамическое определение внешнего поля $\mathbf{h}_{\mathbf{q}}\left(=\left(H_{\mathbf{q}}^{(x)}, h_{\mathbf{q}}^{(y)} h_{\mathbf{q}}^{(z)}\right)\right)$, сопряженного с параметром порядка $\mathbf{M}_{\mathbf{q}}$. Тогда, приняв $h_{\mathbf{q}}^{(\gamma)}=\delta_{\mathbf{q}, 0} \delta_{\gamma, z} h$, имеем

$$
\begin{gathered}
\left\langle\partial \Psi\left(\mathbf{M}_{\mathbf{q}}, \boldsymbol{\zeta}_{\mathbf{q}}\right) / \partial \mathbf{M}_{\mathbf{q}_{0}}^{(\mp)}\right\rangle=M_{\mathbf{q} 0}^{(\mp)}\left(r+A \mathbf{q}_{0}^{2}\right) \\
+\Gamma M_{-\mathbf{q}_{0}}^{(\mp)}\left(\mathbf{M}_{\mathbf{q}_{0}}\right)^{2} \mp \bar{d}\left|\mathbf{q}_{0}\right| M_{-\mathbf{q}_{0}}^{( \pm)}=0, \\
\left\langle T \partial \Psi\left(\mathbf{M}_{\mathbf{q}}, \boldsymbol{\zeta}_{\mathbf{q}}\right) / \partial M_{0}^{(z)}\right\rangle=T M_{0}^{(z)}\left(r+\Gamma\left|M_{\mathbf{q}_{0}}^{(z)}\right|^{2}\right)=h, \\
\left\langle\partial \Psi\left(\mathbf{M}_{\mathbf{q}}, \boldsymbol{\zeta}_{\mathbf{q}}\right) / \partial M_{\mathbf{q}_{0}}^{(z)}\right\rangle=M_{\mathbf{q}_{0}}^{(z)}\left(r+A \mathbf{q}_{0}^{2}\right) \\
+\frac{1}{2} \Gamma M_{-\mathbf{q}_{0}}^{(z)}\left(\mathbf{M}_{\mathbf{q}_{0}}\right)^{2}+\Gamma M_{0}^{(z) 2} M_{\mathbf{q}_{0}}^{(z)}=0 .
\end{gathered}
$$

Здесь, $\bar{d}=x^{2} d$ (см. (3)), а вектор $\mathbf{q}_{0}$ отвечает максимуму модуля неоднородной намагниченности $\left(\left|\mathbf{q}_{0}\right|=\bar{d} / 2 A\right)$,

$$
\begin{gathered}
r=\tau+(6)^{-1} \Gamma\left(x\left|\mathbf{M}_{\mathbf{q}_{0}}\right|^{2}+2 x \mathbf{M}_{0}^{2}+\left\langle\mathbf{M}^{2}\right\rangle\right), \\
\Gamma=\Gamma^{0} \frac{1-\left(\Gamma^{0}\left\langle\mathbf{M}^{2}\right\rangle_{T}\right)^{2}}{1+\left(\Gamma^{0}\left\langle\mathbf{M}^{2}\right\rangle_{T}\right)^{2}},
\end{gathered}
$$

$\left.\left\langle\mathbf{M}^{2}\right\rangle=N_{0}^{-1} \sum_{\boldsymbol{v}}\right\rangle-$ среднеквадратическая амплитуда флуктуаций магнитного момента на узле, включающая в себя как термодинамические флуктуации $\sum_{\mathbf{q}}\left\langle\left|\xi_{\mathbf{q}}-\mathbf{M}_{\mathbf{q}}\right|^{2}\right\rangle$, так и концентрационные $N_{0}^{-1} \sum_{\boldsymbol{v}} \overline{\left(\delta p_{\boldsymbol{v}}\right)}\left|\mathbf{M}_{\boldsymbol{v}}\right|^{2}$.
Ниже температуры $T_{C}$ при $\Gamma^{0}\left\langle\mathbf{M}^{2}\right\rangle<1$ уравнения (5) соответствуют уравнениям магнитного состояния аналогичным получаемым в модели Янсена-Бака $(\Gamma>0)$ :

$$
M_{\mathbf{q}_{0}}^{(z)}=0 \quad M_{\boldsymbol{v}}^{(x)}=M_{S} \cos \left(\mathbf{q}_{0} \boldsymbol{v}\right), \quad M_{\boldsymbol{v}}^{(y)}=M_{S} \sin \left(\mathbf{q}_{0} \boldsymbol{v}\right),
$$

где

$$
M_{S}^{2}=|\Gamma|^{-1}\left(r^{2}+\bar{d}^{2}\left|\mathbf{q}_{0}\right|^{2}\right)^{1 / 2}
$$

В области температур $T>T_{C}$, отвечающих отрицательному значению параметра межмодового взаимодействия $\left(\Gamma<0\right.$, при $\left(\Gamma^{0}\left\langle\mathbf{M}^{2}\right\rangle>1\right)$, имеет место индуцированный спиновыми флуктуациями магнитный переход. При этом получаем, что параметр порядка в решении Янсена-Бака становится равным нулю. Решение для намагниченности узлов теперь содержит начальную фазу $\phi$, величина которой фиксирована в пределах радиуса ферромагнитных корреляций

$$
R_{C}=A^{1 / 2}\left(\tau+(6)^{-1} \Gamma\left(x\left|\mathbf{M}_{\mathbf{q}_{0}}\right|^{2}+2 x \mathbf{M}_{0}^{2}+\left\langle\mathbf{M}^{2}\right\rangle\right)\right)^{-1} .
$$

Тогда в плоскости перпендикулярной оси геликоида (направление волнового вектора сверхструктуры), имеем, что возникают флуктуации магнитной (спиновой) спирали, обусловленные стохастическими флуктуациями начальной фазы,

$$
\begin{gathered}
M_{\boldsymbol{v}}=M_{S} \cos \left(\mathbf{q}_{0} \boldsymbol{v}+\phi\right), \quad M_{\boldsymbol{v}}^{(y)}=M_{S} \sin \left(\mathbf{q}_{0} \boldsymbol{v}+\phi\right) . \\
M_{S}^{2}=|\Gamma|\left(r^{2} \bar{d}^{2}\left|\mathbf{q}_{0}\right|^{2}\right)^{1 / 2}
\end{gathered}
$$

Кроме того получаем, что во внешнем магнитной поле возникает модулированная с вектором геликоида $\mathbf{q}_{0}$ намагниченность вдоль оси $\mathrm{OZ}$

$$
\begin{gathered}
M_{\boldsymbol{v}}^{(z)}=M_{q_{0}}^{(z)} \cos \left(\mathbf{q}_{0} \boldsymbol{v}+\phi\right), \\
\left|M_{\mathbf{q}_{0}}^{(z)}\right|^{2}=\left(M_{0}^{(z)}(h)\right)^{2}-\left[\bar{d}\left|\mathbf{q}_{0}\right| /(4|\Gamma|)\right] .
\end{gathered}
$$

Полученные в лестничном приближении Бразовского [3] для термодинамических флуктуаций с учетом концентрационных флуктуаций, выражения $(8,9)$ соответствуют скирмионным решениям, которые согласуются c $[6,8]$.

\section{3. Анализ в приближение виртуального кристалла}

Рассмотрим стохастические флуктуации модуля локальной намагниченности в модели виртуального кристалла со случайным размещением атомов по узлам кристаллической решетки

$$
\overline{\left(\delta p_{v} \delta p_{\mu}\right)}=x(1-x) \delta_{v, \mu}
$$


Тогда

$$
\begin{gathered}
\left\langle\mathbf{M}^{2}\right\rangle=\left\langle\mathbf{M}^{2}\right\rangle_{T}+x(1-x)\left(\left|\mathbf{M}_{\mathbf{q}_{0}}\right|^{2}+\left(M_{0}^{(z)}\right)^{2}\right), \\
\left\langle\mathbf{M}^{2}\right\rangle_{T}=\left(r+2 \Gamma M_{S}^{2}+X\right)^{-1},
\end{gathered}
$$

где $X=(3 / 5) A q_{C}^{2}$.

Переходя к анализу термодинамических условий существования решений $(8,9)$, следует отметить, что в используемом лестничном приближении Бразовского [3] величина $\left\langle\mathbf{M}^{2}\right\rangle$ не может быть больше единицы. Поэтому значения $|\Gamma|$ ограничены снизу величиной $\bar{d}\left|\mathbf{q}_{0}\right| /(2 A)$, и претерпевает скачкообразное изменение при $T=T_{C}$, что и ведет к скачку энтропии и соответствует переходу первого рода

$$
\begin{aligned}
\Delta S\left(T_{C}\right)= & S\left(T_{C}-0\right)-S\left(T_{C}+0\right) \propto \Gamma\left(T_{C}-0\right) M_{S}^{4}\left(T_{C}\right) \\
& +\left|\Gamma\left(T_{C}+0\right)\right|\left(M_{S}^{2}\left(T_{C}\right)+\left|M_{q_{0}}^{(z)}\left(T_{C}\right)\right|^{2}\right)^{2} .
\end{aligned}
$$

Зависимость $T_{C}$ от однородной намагниченности (однородного магнитного поля), обусловленная перенормировкой концентрационными флуктуациями вершинной части четвертого порядка, определяется уравнением

$$
\begin{aligned}
T_{C}= & T_{C}^{(0)}\left(1+x d\left|q_{0}\right|-2 \Gamma_{x}(1-x) M_{0}^{(z) 2}\right) / \\
& {\left[1+x d\left|\mathbf{q}_{0}\right|-\Gamma x(1-x) M_{0}^{(z) 2}-\Gamma^{0}\right] }
\end{aligned}
$$

и имеет минимум при

$$
M_{0}^{(z) 2}=\Gamma^{0} M_{S}^{2}\left[\Gamma^{0} x(1-x)+1-\Gamma^{0} / 2\right]^{-1} .
$$

Этот минимум соответствует минимальному значению $T_{C}$ на линии $T_{C}(h)$, ограничивающей скирмионную фазу на $(h-T)$-диаграмме, экспериментально установленной в работе [7]. При устремлении $x$ к нулю или единице $T_{C}>T_{C}^{(0)}$, a $\left(M_{\mathbf{q})_{0}}^{(z)}(h)\right)^{2}$ обращается в ноль.

Согласно соотношениями $(8,9)$, границы А-фазы характеризуются наличием ненулевого модуля локальной намагниченности (которая согласно (8) стохастически флуктуирует в пространстве)

$$
\begin{aligned}
M_{S} & =M_{S}(0) \\
& \times\left(1-\left\langle\mathbf{M}^{2}\right\rangle_{T} /\left(M_{S}^{2}(0)+x(1-x)\left(M_{0}^{(z)}(h)\right)^{2}\right)\right)^{1 / 2} .
\end{aligned}
$$

Из (14) следует, что локальная намагниченность исчезает вследствие тепловых флуктуаций при температуре

$$
T_{S} / T_{C}=1+x(1-x) d\left|\mathbf{q}_{0}\right|+2\left(M_{0}^{(z)}(h)\right)^{2} x(1-x),
$$

величина которой претерпевает существенное изменение в зависимости от концентрационных флуктуаций. Так в случае нестехиометрического моносилициде марганца с дефицитом марганца $10 \%(x=0.9)$ согласно (13) получаем увеличение максимальной ширины температурного интервала $\left(T_{S}(h)-T_{C}(h)\right)$ по сравнению со стехиометрическим составом примерно на порядок: от значения 1.2 К в случае $\mathrm{MnSi}$ до значения $12.7 \mathrm{~K}$ [7].

\section{4. Заключение}

Таким образом, при индуцированном флуктуациями магнитном фазовом переходе в геликоидальном ферромагнетике с ДМ-взаимодействием наличие среднеквадратических отклонений заполнения узлов магнитными атомами оказывают значительное влияние на формирование скирмионных состояний. Это влияние связано с тем, что концентрационные эффекты сохраняют локальную намагниченность непосредственно выше температуры индуцированного флуктуациями фазового перехода первого рода. При этом термодинамические спиновые флуктуации, взаимодействие которых индуцирует переход в точке $T_{C}$, приводят к подавлению локальной намагниченности при температуре $T_{S}$. Возникающий в интервале температур от $T_{C}$ до $T_{s}$ магнитный порядок характеризуется флуктуациями спиновой спирали, и частичным упорядочением вдоль оси параллельной внешнему магнитному полю синусоидальной волны спиновой плотности.

В развитой нами модели концентрационные флуктуации аналогичны продольным флуктуациям магнитных моментов на узле в микроскопической модели. Согласно Монте-Карло моделированию $[5,6]$ учет именно продольных флуктуаций является особо важным для объяснения особенностей магнитного рассеяния нейтронов в области А-фазы $[7,8]$. Здесь мы получаем, что концентрационные флуктуации, приводящие к заметной перенормировке модуля локальной намагниченности, могут заметно увеличивать температурный интервал А-фазы.

В дальнейшем требуется микроскопическое обоснование развитой модели в части определения параметров электронной структуры нестехиометрических составов $\mathrm{MnSi}$ на основе первопринципных расчетов (в частности $\left.\Gamma^{0}\left\langle\mathbf{M}^{2}\right\rangle\right)$. Значительный эффект отклонений от стехиометрического состава $\mathrm{MnSi}$ на локальную намагниченность может быть связан с возникновением флуктуаций внутриатомного кулоновского взаимодействия на узле. Действительно, согласно LSDA+U+SO-расчетам [9] $U=0.9$ эВ для узла занятого марганцем и $U=0$ для узла занятого кремнием $\left(p_{v}=0\right)$.

\section{Финансирование}

Результаты были получены в рамках задания министерства образования и науки Российской Федерации, контракт 3.9521.2017/8.9

\section{Конфликт интересов}

Авторы заявляют, что у них нет конфликта интересов.

\section{Список литературы}

[1] P. Bak, M.H. Jensen. J. Phys. C 12, L881 (1980).

[2] В.П. Минеев. УФН 187, 129 (2017).

[3] С.В. Бразовский. ЖЭТФ 68, 175 (1975). 
[4] M. Janoschek, M. Garst, A. Bauer, P. Krautscheid, R. Georgii, P. Böni, C. Pfleiderer. Phys. Rev. B 87, 134407 (2013).

[5] S. Buhrandt, L. Fritz. Phys. Rev. B 88, 195137 (2013).

[6] A.M. Belemuk, S.M. Stishov. Phys. Rev. B 97, 144419 (2018).

[7] N. Potapova, V. Dyadkin, E. Moskvin, H. Eckerlebe, D. Menzel, S. Grigoriev. Phys. Rev. B 86, 060406(R) (2012).

[8] Y. Dovzhenko, F. Casola, S. Schlotter, T.X. Zhou, F. Büttner, R.L. Walsworth, G.S.D. Beach, A. Yacoby. Nature Communications 9, 2712 (2018).

[9] A.A. Povzner, A.G. Volkov, T.A. Nogovitsyna. Physica B 536, 408 (2018).

Редактор Ю.Э. Китаев 